\section{A Climiral $\mathfrak{l}$ lerture}

\section{CANCER OF THE RECTUM.} BY

JAMES SWAIN, M.S., M.D.Lond., F.R.C.S.EnG., PROFESSOR OF SURGERY IN THE UNIVERSITY OF BRISTOL; SURGEON TO THE BRISTOL ROYAL INFIRMARY.

In dealing with the extirpation of carcinomatous growths in various parts of the body the modern tendency is to enlarge the area of operation in order that the lymphatic supply of the part affected may be widely removed with the primary growth. I remember the time when the axillary glands were only removed in cases of cancer of the breast when they were palpably enlarged, but now we remove the pectoral and axillary lymphatic areas in every case. In cancer of the tongue, too, it is only in compara. tively recent times that we have aropted the removal of the lymphatics of the neck as a routine measure, and in this, as in the case of cancer of the breast, the wider operation has resulted in a diminished tendency to that recur rence which all patients afflicted with cancer have such good reason to dread.

Many of the operations for cancer of the rectum do not allow of a sufficient removal of the lymphatic area in the neighbourhood of the growth, and recurrence of the mischief is so common that Bergmann ${ }^{1}$ considers it probable that only "about one-fourth or one fifth of the patients operated upon for cancer of the rectum can be permanently freed trom their disease." This deplorable state of affairs is only likely to be improved when it becomes the rule to operate in such a manner as to permit of a more extensive removal of the lymphatics, as in the case of operations for cancer of the breast and tongue, and it is in order that we may consider how this may be best accomplished that I have brought this subject before you to-day-exemplified by a case which will serve to illustrate some points in the diagnosis and treatment of cancer of the rectum.

Mrs. W., aged 56 years, was admitted under my care for pain and difficulty in defaecation For twelve months previously she had complained of a gradually increasing constipation, and aperients were constantly necessary. The stools frequently contained blood, and there was also a more or less continuous blood-stained discharge. There was rectal tenesmus, and at times the motions were "loose and frothy." There had been loss of flesh and a general feeling of languor. For the past three months these symptoms had increased in severity, and there had been considerable sacral pain, especially after walking. The patient was pallid and somewhat thin. No tumour could be felt in the abdomen. Per rectum a hard irregular growth could be felt projecting in to the lumen of the gut about $2 \frac{1}{2}$ in. from the anus. The rectum was invaginated br the growth, and on palpation resembled the cervix uteri. The lumen of the gut at the situation of the tumour would just admit the forefinger. The growth was movable, and there was no involvement of the vagina or other tissues in the neighbourhood. No enlarged glands could be felt. After a period of purgation and enemata to thoroughly empty the lower bowel, the patient was placed in the Trendelenburg posture, and a median abajominal incision was made from just below the umbilicus to the pubes. A double ligature was placed on the sigmoid flexure near its centre, and the bowel divided between the two ligatures. The upper end was tied up in gauze to prevent infection, and the lower end was invaginated into the bowel below and the lower end was invaginated into the bowel below
by means of a purse-string suture. An incision was then made through the peritoneum on either side of the rectumn, joined below by an incision through the peritoneum of the pouch of Douglas. The flaps of peritoneum were turned outwards, and the superior haemorrhoidal vessels were tied near the top of the sacrum. The lower part of the sigmoid and the rectum were then freed from all surrounding connexions as far as the insertion of the levatores ani, the mesorectum and all its contained lymphatic glands being turned out of the hollow of the sacrum with the rectum by forcibly detaching it from the bone by gauze. The parts thus set free were crowded down into the pelvis, and the cut portions of the peritoneum were approximated. The patient was then placed in the lithotomy position, a circular incision was made round the anus and extended backwards to the coccyx in the mid line. Through this incision the lower part of the rectum was freed, and, after division of the lower part of the rectum was freed, and, after division of the levatores ani, the whole rectum, with the mesorectum and glands and part of the sigmoid flexure, were delivered through inserted, and the riost A rubber drain and was closed. The upper end of the sigmoid (which had been tied up in gauze) was then brought through an opening made by blunt division of the muscles in the left iliac fossa near the anterior superior iliac spine. The ligature round the bowel was removed and a Paul's tube tied into the lumen, the gut being sewn to the adjacent peritoneum. The median abdominal incision was completely closed. The operation was not followed by any marked shock, convalescence was uninterrupted, and the patient left the Infirmary on the twenty-sixth day after operation with the abdominal and perineal wounds quite healed, the colotomy wound in a healthy condition, and the bowel acting satisfactorily. On microscopical examination the growth proved to be of the ordinary type of adeno-carcinoma.

The symptoms of which this patient complained may be regarded as typical of cancer of the rectum, but for a long time they were regarded as due to piles. This is perhaps the most common mistake, owing to the fact that haemorrhage and rectal discomfort are common to piles and cancer, but if we are to reduce the present high rate of recurrence in the latter, it is important that the cases should be diagnosed correctly as early as possible.

The early stages of cancer of the rectum are frequently unaccompanied by any marked symptoms, and the insidiousness of the disease is well known. Pain occurs in all cases of rectal cancer sooner or later, and towards the end may be very severe, but in the early stage it is, in my experience, often absent. Haemorrhage is perhaps a more common symptom, but this may be absent until the disease is well established. I bave recently had under my care, in private, two cases which emphasize the difficulty of diagnosis in these cases unless a proper examination is made in all cases of rectal trouble, and this is the only way in which you can prevent yourself from falling into serious error. The one was a gentleman who had slight constipation and bleeding, which he attributed as usual to piles; but noticing on one occasion a little more blood than usual he was wise enough to consult his medical attendant, and the latter fortunately made a rectal examination and discovered the cancer of the rectum. The patient looked perfectly well, and felt so, and yet he had a fairly advanced condition of the disease, though I was able to perform on him an operation similar to the one above referred to. The other case was in a patient aged 60 who had a slight rectal discomfort but had had no pain haemorrhage, diarrhoea. or constipation, and yet be had an advanced condition of cancer of the rectum associated with such widespread glandnlar involvement that a radical operation was impossible. Ulceration and stenosis occur relatively early, and then we get the passage of blood. stained mueus, foul smelling discharge and diarrhoea, alternating with constipation. The last fact is to be borne in mind, and all cases complaining of an uncontrollable diarrhoea should be subjected to rectal examination.

Our aim should be to arrive at a diagnosis sufficiently early in the case to allow of a radical operation with a good prospect of ridding the patient of the mischief. In all cases of definite rectal trouble a digital examination should be.insisted on, and it is sometimes found that a growth which cannot otherwise be reached may be brought to the examining finger by getting the patient to strain downwards in the standing position. If no growth is discovered and cancer is suspected a further examination should be made under an anaesthetic, and with the aid of the sigmoidoscope if necessary.

Although most cases of cancer of the rectum commence after 40 years of age, the disease occurs with even greater virulence in younger people. I have excised the rectum for cancer in a patient aged 21 years, and other observers have operated for this condition at a still earlier age.

The later characters of the growth have been described in the case under discussion; earlier in the disease the hard nodular margin of the growth infiltrating the tissues around and the smoothness of the unaffected mucous mem. brane below leave no doubt as to the character of the mischief, even though the patient looks quite healthy and has suffered little discomfort.

When the diagnosis bas been established we have to decide whether the case is suitable for a radical operation. There was no difficulty in doing this in the case I have narrated, where the growth was movable and nonadherent, though a slight involvement of the vaginal walls need not deter one from operation. But where the bladder is involved, or where there are extensive adhesions to the sacrum, a radical operation should not be recommended, as recurrence would certainly take place. When there is sufficient obstruction to cause an accumulation of faec $\mathrm{S}$ above the growth, the amount of fixation often appears to be more than is actually the case. You should not, 
therefore, hastily decide that a case is unfit for the radical operation until the bowel has been thoroughly emptied by means of enemata and purgatives. It is not uncommon to find that with the removal of the accumulation the growth is much more limited than it at first seemed to be.

Having decided to perform a radical operation, it is highly desirable that the bowel should be empty beforehand, and though it may take the greater part of a week to ensure this condition by means of enemata and aperients, the time is well spent. A loaded intestine is a source of danger at the time of operation, and, moreover, patients suffering from the effects of absorption of intestinal toxins are less able to bear the shock of a severe operation. In late cases, where the patient has a definite chronic obstruction, it is occasionally necessary to perform a preliminary colotomy, in order that the bowel may be relieved of its germ-laden contents before the radical uperation is procoeded with. It should, however, be cur constant aim to operate at an early stage of the disease, before any marked obstruction has supervened.

There are several ways of attacking a rectal carcinoma. Let me first briefly enumerate some of these, and then point out to you the reasons why I chose that method of operation which was adopted in the case which forms the basis of this lecture.

A growth situated, as this one was, about $2 \frac{1}{2}$ in. from the anus might have been removed by the sacrococcygeal route (Kraske's operation), by the abdomino-anal route, in which the sigmoid flexure is brought down to the anus after the whole rectum has been excised, or by the abdomino-perineal route, in which the whole rectumincluding the sphincter-is excised and an artificial anus made in the left iliac fossa or other part of the lower abdomen. It was this last method which was adopted in the case above quoted.

Apart from the fact that those of us who are familiar with abdominal operations find it easier to excise the rectum from above, it is clear that a wider removal of the rectum and the lymphatic glands of the pelvis is possible through an abdominal incision. That this more extensive operation is necessary is shown by the unsatisfactory resuits of the sacro-coccygeal (Kraske) operation. We cannot be content with a method that is not only attended by a fairly high mortality, but which also is followed by recurrence of the disease in a large proportion of cases. The average mortality of Kraske's operation in the hands of twelve well known surgeons appears to be over 20 per cent., and the permanent cures after rectal excision, as shown by the average results of nine leading operators, is under 16 per cent. ${ }^{2}$

These results leave much to be desired, and I believe that by the adoption of some form of abdominal operation in all cases of cancer of the rectum (except, perhaps, those which are situated quite low down near the anus) we may ultimately succeed in placing the operative treatment of this disease on a satisfactory basis.

Having explained the reasons for preferring one of the forms of abdominal operation, let us see why the abdomino. perineal route was chosen instead of the abdomino-anal; or, in other words, why was an artificial anus made in the left iliac fossa instead of bringing the divided sigmoid flexure down to the anus? The abjomino-anal operation is certainly preferable if the patient has sphincteric control; and in this case, as in the majority of patients, it would have been quite possible to bring the divided sigmoid down to the anus; but it appeared doubtful whether the growth was not too low to run the risk of pourrence by leaving any part of the rectum, and, in any case, it seemed certain that the necessary incisions for freeing the lower part of the gut must result in the division of the nerve supply of the external sphincter, which would, therefore, be functionless. If sphincteric control cannot be obtained, it is better to make an artificial anus in thy lower abdomen.

Some time ago I performed the abdomino-anal operation upon a lady in whom I was able to preserve the sphincters, but (probably owing to nerve division), unfortunately, control was lost. The resulting incontinence was so troublesome that I yielded to her desire to perform a colotomy some months after the operation for excision of the rectum. This was found to be much more manageable, and probably most patients would prefer an artificial anus above, rather than an anus over which they had no control in the normal situation. On the other hand, it is highly desirable to preserve the sphincters and bring the divided sigmoid down to the anus in all cases where it is possible so to do.

There is one other question which may arise in your minds-namely, the reason why the artificial anus was made in the left iliac fossa rather than by anchoring the divided sigmoid flexure in some part of the median abdominal incision. In the performance of an ordinary sig. moidostomy an incision through the left rectus near the median line is sometimes deliberately chosen with advantage, and I have found such an artificial anus very satisfactory; but after excision of the rectum it is undesirable to risk the fouling of the comparatively large centra incision by the necessary escape of faeces, and, therefore it is better to make the artificial anus through a separate opening in the left iliac fossa. In this way contamination of the median incision can be avoided, and healing by first intention can thus be generally assured.

The operation of excision of the rectum for cancer which I have been advocating through an abdominal incision has not yet been generally adopted, and it is too early to com. pare its mortality and the freedom from recurrence of the disease afterwards with other methods. In the sacrococcygeal route it is a well.known fact that the higher the disease is situated in the rectum the greater the mortality. Again quoting Bergmann, ${ }^{8}$ who refers to Rave's statistics of 335 cases of rectal carcinoma treated by operation in various clinics, he says:

The higher tumours were almost all operated upon by Kraske's method, with a mortality of 23 per cent. . . For higb-seated tumours it gave a mortality of 33 per cent. in women and 19 per cent. in men. This higher mortality in women may be explained by the fact that the tumours easier of access, were operated upon perineally in women.

We might, therefore, presume that the abdominal operation would probably be attended by a high mortality, but we must remember that with new methods of treatment the mortality is generally higher than when the principles of the operation are better understood. The mortality of Kraske's operation is said to have been from 30 to 50 per cent. at first, but this was diminished when better means for the prevention of sepsis were adopted. I have elsewhere ${ }^{4}$ shown that resection of the large bowel for carcinoma is fairly well borne even by those of advanced years, and I have not found, so far, any excessive amount of shock in the operation of excision of the rectum by the abdominal route.

With our present means of preventing infection of the operation area, 1 do not think the mortality of the abdominal route will be found unduly high in experienced hands; and the greater opportunity we have, by its adoption, of examining the growth (and deciding for or against the desirability of excision) and the more thorough removal of the lymphatics with the primary growth which this method allows, will probably ensure for it the chief place in the various radical operations for carcinoma of the rectum.

1 V. Bergmann, A System of Practical Surgery, vol. v, p. $172 .{ }^{2}$ Gant, quoted in Kelly and Noble's Gynecology and Abdominal Surgery MEDIOAL JoURnaL, January 10th, 1903.

THE lectures and discussions of the Child Study Society were resumed at the house of the Royal Sanitary Insti. tute, 90, Buckingham Palace Road, S.W., on Thursday last, when Dr. R. Langdon-Down gave an address on the social relation of the child. Next Thursday Dr. J. Lionel Tayler will speak on types of children, their observation, and nurture. On April 7th Dr. T. B. Hyslop will give a lecture on subconsciousness, and on April 21st Dr. C. A. Mercier will introduce a discussion of the aims of education. The meetings begin at 7.30 .

THE late Mr. John Wortley Axe, Chief Veterinary Inspector to the Surrey County Council, in addition to making certain other dispositions for the benefit of the Royal College of Veterinary Surgeons and its old students, bequeathed $£ 400$ to its council to defray the expense of painting full-length portraits of Professors Spooner, Summers, Varnell, and Brown, to be hung in the council chamber of the college in recognition of their great services to veterinary science. 